\title{
CONDICIONES METEOROLÓGICAS Y DINÁMICA VEGETAL DEL ECOSISTEMA COSTERO LOMAS DE ATIQUIPA (CARAVELÍ - AREQUIPA) EN EL SUR DEL PERÚ
}

\section{METEOROLOGICAL CONDITIONS AND VEGETAL DYNAMICS OF THE ATIQUIPA LOMAS (CARAVELÍ - AREQUIPA) COASTAL ECOSYSTEM IN THE SOUTH OF PERÚ}

\author{
Diego A. Sotomayor Melo ${ }^{1}$ y Percy Jiménez Milón ${ }^{2}$
}

\begin{abstract}
Resumen
Los ecosistemas costeros de Lomas están constituidos por comunidades vegetales desarrollándose bajo influencia de neblinas invernales. Para explorar este enunciado se determinó la relación entre las condiciones meteorológicas y la dinámica vegetal en las Lomas de Atiquipa, cerros Barrera y Lagunillas (15\%46' LS y 74²2' LO, a 800 msnm aprox) entre Febrero 2005 y Febrero 2006. En cada cerro se instalaron 45 parcelas de 0.5 x $0.5 \mathrm{~m}$; evaluándose mensualmente por parcela el número de especies e individuos por especie (plántulas y adultos), cobertura por especie, cobertura de hojarasca y estado fenológico. Además se calcularon los índices de Simpson y Shannon-Wiener. Se registró mensualmente temperatura media, captación de neblina y precipitación natural. Con estos datos se realizaron correlaciones lineales entre los parámetros ambientales (3) y de vegetación (10). Se encontraron varias relaciones significativas $(\mathrm{p}<0.05)$; neblina con 5 relaciones significativas: directa con el número total de individuos en Barrera $(\mathrm{r}=0.79)$, la emergencia de plántulas en Barrera $(\mathrm{r}=0.79)$ y Lagunillas $(\mathrm{r}=0.77)$, y con el predominio en Barrera $(\mathrm{r}=0.65)$, e inversa con la diversidad de Shannon - Wiener en Barrera $(\mathrm{r}=$ 0.76). Existió relación inversa entre temperatura y predominio en la comunidad (Barrera $\mathrm{r}=-0.61$; Lagunillas $\mathrm{r}=-0.59)$. La precipitación natural no presentó relaciones significativas, aunque puede sugerirse un efecto acumulativo entre Julio y Agosto que desencadenó emergencia de plántulas en Setiembre. Mayores estudios de estas relaciones son necesarios, especialmente en un escenario de cambio climático que afectaría el régimen de humedad en estos frágiles ecosistemas.

Palabras clave: Dinámica vegetal, condiciones meteorológicas, relación clima - vegetación, Lomas costeras, Atiquipa, Sur de Perú, desiertos, zonas áridas Perú
\end{abstract}

\begin{abstract}
Coastal ecosystems of Lomas are constituted by plant communities developing under winter fog influences. In order to explore this issue we determined the relationship between vegetation dynamics and meteorological conditions at Atiquipa Lomas, Barrera and Lagunillas hills (15 46' LS y $74^{\circ} 22^{\prime}$ LW, 800 m.a.s.l. aprox.) from February 2005 to February 2006. Forty-five 0,5 x 0,5 m plots were installed in each hill, with monthly plot evaluations of species number, individuals per species (seedlings and adults), species cover, fallen leaves cover and phenological status. Simpson and Shannon-Wiener indexes were calculated. Natural precipitation, fog collection and medium temperature were also monthly registered. With these data we ran lineal correlations between environment parameters (3) and vegetation (10). Several significant relationships $(\mathrm{p}<0.05)$ were found, fog had 5 significant relationships: direct with total individuals in Barrera $(\mathrm{r}=0.79)$, with seedling emergence in Barrera $(\mathrm{r}=0.79)$ and Lagunillas $(\mathrm{r}=0.77)$, and with Simpson's predominance in Barrera $(\mathrm{r}=0.65)$, and inverse with Shannon-Wiener's diversity in Barrera $(\mathrm{r}=-$ 0.76). There was an inverse relationship between temperature and predominance in the community (Barrera $\mathrm{r}=-0.61$; Lagunillas $\mathrm{r}=-0.59$ ). Natural precipitation did not show any significant relationships, although a cumulative effect between July and August which unleashed seedling emergence in September can be suggested. More research concerning these relationships is necessary, especially in a climate change scenario which could affect moisture regimes in these dynamic fragile ecosystems.

Key words: Vegetal dynamics, meteorological conditions, weather-vegetation relationships, Coastal Lomas, Atiquipa, Southern Peru, deserts, Peruvian arid zones
\end{abstract}




\section{Introducción}

El Desierto costero Peruano - Chileno representa un cinturón continuo muy árido de más de $3500 \mathrm{~km}$ a lo largo de un estrecho territorio ubicado en la vertiente occidental de la Cordillera de los Andes (Rundel et al., 1991). Dentro de este desierto se presentan los ecosistemas de Lomas, referidos como "praderas de vegetación en medio del desierto" por Ono (1986), cuya distribución es exclusiva para Sudamérica, desde los cerros Campana y Cabezón en Trujillo - Perú ( $\left.8^{\circ} \mathrm{LS}\right)$ hasta Huasco y Coquimbo en Chile $\left(30^{\circ} \mathrm{LS}\right)$.

En invierno la Corriente Peruana contribuye a formar un manto neblinoso, usualmente entre Junio y Setiembre, el mismo que se condensa y precipita lentamente (Ferreyra, 1986). Este manto neblinoso en zonas colinosas y de pendiente abrupta, es interceptado y genera una zona húmeda que permite el desarrollo de formaciones vegetales llamadas Lomas (Rundel et al., 1991).

Las Lomas son unidades fitogeográficas periódicas que generalmente contienen un número elevado de géneros y especies endémicas (Mostacero et al., 1996). El 42\% de su flora está conformada por especies endémicas (Müller, 1985), dentro de las cuales destacan las pertenecientes a los géneros Mathewsia, Palaua, Weberbauerella, Domeykoa y Nolana, entre otras (Ferreyra, 1986).

Estas comunidades vegetales abarcan en el Perú solo $2000 \mathrm{~km}^{2}$ y en Chile

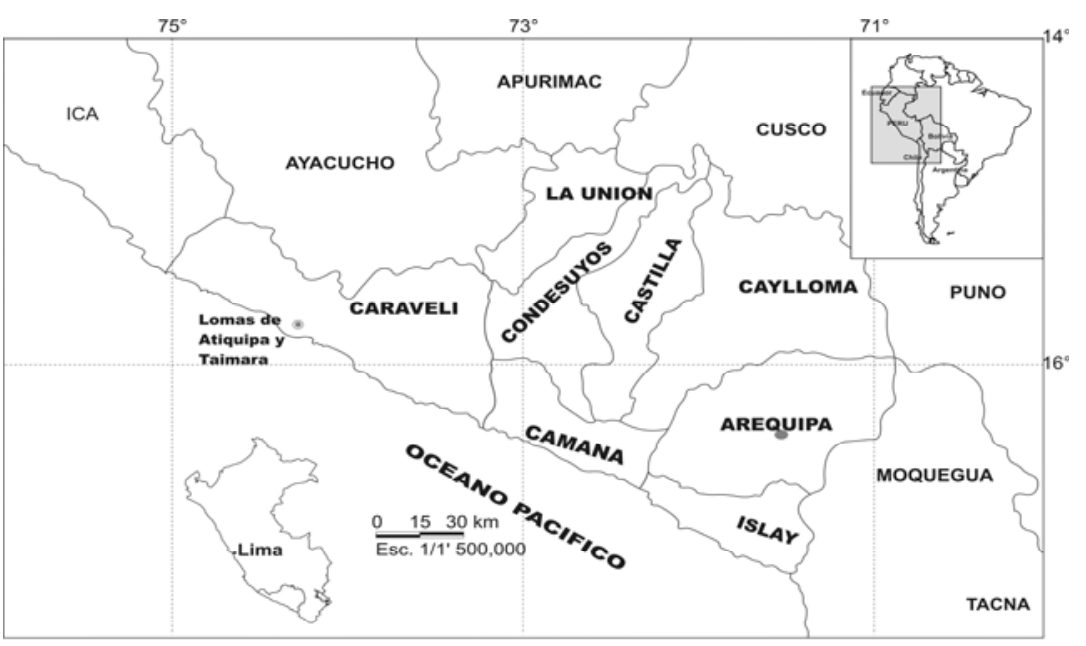

Figura 1. Mapa de ubicación de la zona de estudio Atiquipa - Caravelí, Arequipa. una menor extensión (Mostacero et al., 1996); pero, según se sabe ocuparon más de $15000 \mathrm{~km}^{2}$ hace 5 siglos cuando servían de sustento a centros poblados importantes (Engel, 1981). Las causas de esta dramática disminución son el sobrepastoreo y la deforestación (Mostacero et al., 1996).

Dentro de estas formaciones vegetales, las Lomas de Atiquipa, representan un ecosistema muy importante, debido a que tienen un área estimada de 22800 ha (Canziani \& Mujica, 1997), la más grande dentro de este tipo de formaciones; y por su estructura vegetal que ofrece una gran variedad de hábitats (Villegas et al., 2004).

En este estudio se investiga una de las relaciones más importantes de los ecosistemas de Lomas: la relación clima - vegetación, trabajando con la hipótesis de que existe relación significativa entre las condiciones meteorológicas y la dinámica vegetal.

\section{Material y métodos}

Descripción de la zona de estudio

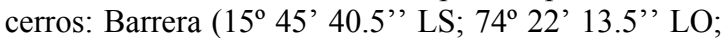
798 msnm) y Lagunillas (15' 46' 00.1' ' LS; 74 $22^{\prime}$ ' 26.4' LO; 805 msnm); ubicados en las Lomas de Atiquipa - Caravelí, Arequipa (Figura 1). Pendiente y exposición en ambos cerros es similar, tratándose de lugares prácticamente planos de exposición sureste. Ambos cerros se encuentran en el área de protección estricta que la Comunidad Campesina de Atiquipa ha destinado para conservar los recursos naturales de las Lomas evitando el ingreso de animales grandes, especialmente herbívoros domésticos. Antes del 2001, cuando empieza la protección, toda esta área estuvo sometida a fuerte pastoreo y tala indiscriminada de árboles.

La zona corresponde al área colinosa propia de la

Cordillera de la Costa, presente entre los 500 y 1297 msnm, con mayor humedad que el resto de las Lomas, pastos y vegetación arbustiva (Canziani \& Mujica, 1997). De acuerdo a ONERN (1976) pertenece al desierto perárido - templado cálido $(\mathrm{dp}-\mathrm{Tc})$.

Caracterización de las condiciones meteorológicas

Durante el periodo evaluado (Febrero 2005 Febrero 2006) la temperatura media fue de $15.2{ }^{\circ} \mathrm{C}$; fluctuando entre $19.7^{\circ} \mathrm{C}$ en Febrero del 2005 y 2006, y $11.8{ }^{\circ} \mathrm{C}$ en Agosto 2005. La precipitación total para este periodo fue de $82.7 \mathrm{~mm}$, siendo Setiembre 2005 el mes de mayor precipitación con $24.9 \mathrm{~mm}$. En Febrero 2005, Marzo 2005, Abril 2005, Junio 2005 y Febrero 2006 no se registró precipitaciones. Estos datos fueron proporcionados por el Proyecto PER/01/G35, registrados por la Estación Meteorológica automática Vantage Pro2 instalada en cerro Barrera a 795 msnm. Debido a que la Estación fue instalada en Agosto 2005, no se disponen datos de temperatura para la zona de estudio, por lo que estos
Este estudio se realizó en la parte superior de dos 
se aproximan utilizando los mismos datos de la Estación para 2006; los datos de precipitación para el periodo previo a la instalación de la Estación automática fueron registrados en un pluviómetro ubicado en cerro Barrera. En Setiembre 2005 se registró la mayor cantidad de captación de neblina con $66.51 / \mathrm{m}^{2} /$ día, mientras que Febrero 2006 registró la menor cantidad con $1.7 \mathrm{1} / \mathrm{m}^{2} /$ día (datos obtenidos con un neblinómetro estándar localizado en el cerro Lloque a $980 \mathrm{msnm}$ ). Los datos meteorológicos proporcionados por el Servicio Nacional de Meteorología e Hidrología (SENAMHI) para Punta Atico (16 $\left.{ }^{\circ} 13^{\prime} \mathrm{LS} ; 7^{\circ} 41^{\prime} \mathrm{LO} ; 20 \mathrm{msnm}\right)$ indican un periodo extremadamente seco $(2.5 \mathrm{~mm}$ de precipitación), que no coincide con lo ocurrido en Atiquipa durante este mismo periodo, sin embargo serán considerados en parte debido a que la información de Diciembre 2004 revela una precipitación significativa $(24.0 \mathrm{~mm})$ en Atico que tendría relación con el estado de la vegetación en Atiquipa al inicio del estudio.

Atiquipa mostró una temporada húmeda y fría entre Julio 2005 y Noviembre 2005 con precipitación total de $60 \mathrm{~mm}$, un promedio de $40.91 / \mathrm{m}^{2} /$ día de captación de neblina y con temperatura promedio de $12.8^{\circ} \mathrm{C}$; y dos temporadas secas y cálidas, la primera entre Febrero 2005 y Junio 2005 con precipitación de $10 \mathrm{~mm}, 8.6 \mathrm{l} / \mathrm{m}^{2} /$ día de captación de neblina promedio y $16.2{ }^{\circ} \mathrm{C}$ de temperatura promedio; y la segunda entre Diciembre 2005 y Febrero 2006 con precipitación de $12.7 \mathrm{~mm}, 8.1 \mathrm{l} / \mathrm{m}^{2} /$ día de captación de neblina y 17.5 ${ }^{\circ} \mathrm{C}$ de temperatura promedio (Figura 2).

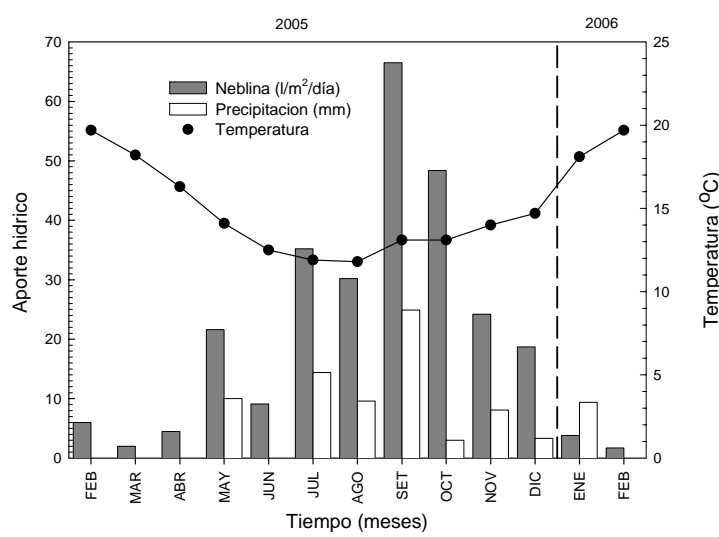

Figura 2. Registros mensuales de temperatura media, precipitación y captación de neblina entre Febrero 2005 y Febrero 2006 para la zona de estudio. Datos proporcionados por el Proyecto PER/01/G35.

\section{Colecta y determinación de especies}

Se realizaron colectas botánicas principalmente en la evaluación inicial (Febrero 2005), recogiéndose preferentemente a aquellos individuos de la misma especie de los que se presentaban dentro de las parcelas, evitando disminuir la población de cada especie dentro de las mismas. Las especies que fueron apareciendo durante el periodo evaluado se marcaron hasta el momento oportuno para su determinación. Las muestras se determinaron en el Herbarium Areqvipense (HUSA).

\section{Diseño de muestreo}

Se siguieron las recomendaciones de Braun Blanquet (1979) y BOLFOR et al. (2000), incluyendo la propuesta de McCook (1994) al trabajar a escalas espaciales pequeñas y con ensambles simples de especies para un mejor entendimiento del ecosistema. La unidad muestral corresponde a una parcela cuadrada de $0.5 \mathrm{~m}$ de lado $(0.5 \times 0.5)$. En cada cerro se instalaron 45 parcelas (90 en total) separadas entre sí por $1 \mathrm{~m}$, las parcelas que se encontraron hacia el borde tuvieron una separación del mismo de $2 \mathrm{~m}$. El terreno de estudio fue de forma rectangular, con 5 parcelas en su ancho y 9 parcelas en su largo.

Evaluaciones realizadas

Se evaluó a principios de cada mes durante 12 meses (doce evaluaciones y una inicial), en donde se anotaron las siguientes características de las parcelas:

a. Número de especies y número de individuos presentes. Conteo directo o en caso de numerosos individuos un aproximado. Además, se registró el número de plántulas e individuos adultos.

b. Cobertura y tamaño aproximado de las especies presentes. Se proyectó sobre el sustrato el conjunto del sistema de vástagos de todos los individuos de la especie y después se determinó la superficie cubierta (Braun Blanquet, 1979).

c. Estado fenológico. Es el estado fenológico dominante en cada especie al momento de hacer la evaluación: plántula, vegetativo, floración, floración - fructificación, fructificación $\mathrm{y}$ senescencia.

d. Hojarasca. Porcentaje de suelo cubierto por hojarasca.

\section{Análisis de datos}

Para analizar el cambio estructural de las comunidades se calcularon dos índices: el índice de Simpson $\left(\mathrm{D}=\Sigma \mathrm{pi}^{2}\right)$, y el índice de Shannon - Wiener $\left(H^{\prime}=-\Sigma\right.$ pi $\left(\log _{2}\right.$ pi $)$ ) (Moreno, 2001); estos valores aparecen en el Anexo 1. La dinámica vegetal estuvo representada por 10 parámetros: 1) número total de individuos, 2) cobertura vegetal, 3) número de individuos adultos, 4) cobertura vegetal de individuos adultos, 5) número de plántulas, 6) cobertura vegetal de plántulas, 7) número de especies, 8) índice de Simpson, 9) índice de Shannon - Wiener y 10) hojarasca. Estos 10 parámetros fueron correlacionados con la captación de neblina, la precipitación natural y la temperatura media promedio registradas entre Febrero 2005 y Febrero 2006 en la zona de estudio. Los valores de $\mathrm{p}<0.05$ fueron considerados como significativos; y las correlaciones (valor de r) entre $0 \mathrm{y}$ 0.3 son malas, entre 0.3 y 0.5 regulares, entre 0.5 y 0.8 
buenas y por encima de 0.8 muy buenas. Se utilizó el software STATISTICA versión 6.0 (StatSoft, 2001).

\section{Resultados}

Listado florístico y comunidades vegetales

Se registraron un total de 21 especies de plantas con flores (Tabla 1), de las cuales 8 estuvieron presentes en ambos cerros. En Barrera se presentaron 14 especies, mientras que en Lagunillas hubo 15 especies. Cerro Barrera estuvo representado por una comunidad perenne donde las especies dominantes fueron Senecio mollendoensis, un arbusto endémico, y Nicotiana paniculata, un sufrútice endémico; mientras que Lagunillas tuvo una comunidad estacional representada fundamentalmente por la terófito nativa Fuertisimalva peruviana, y en segundo orden por la hierba anual ampliamente introducida Portulaca oleracea.

Tabla 1. Listado de las especies encontradas en las parcelas instaladas en los cerros Barrera y Lagunillas de las Lomas de Atiquipa entre Febrero 2005 y Febrero 2006.

\begin{tabular}{|c|c|c|}
\hline Especie & Barrera & Lagunillas \\
\hline 1. Portulaca oleracea L. & $\mathrm{X}$ & $\mathrm{X}$ \\
\hline $\begin{array}{l}\text { 2. Fuertisimalva peruviana (L.) } \\
\text { Fryxell }\end{array}$ & $\mathrm{X}$ & $\mathrm{X}$ \\
\hline 3. Senecio mollendoensis Cabrera & $X$ & $X$ \\
\hline $\begin{array}{l}\text { 4. Cucumis dipsaceus Ehrenberg ex } \\
\text { Spach }\end{array}$ & & $\mathrm{X}$ \\
\hline 5. Nicotiana paniculata $\mathrm{L}$. & $\mathrm{X}$ & $\mathrm{X}$ \\
\hline 6. Nicandra physalodes (L.) Gaertner & & $\mathrm{X}$ \\
\hline 7. Nolana coronata R. \& P. & & $\mathrm{X}$ \\
\hline 8. Solanum sp. & $\mathrm{X}$ & $\mathrm{X}$ \\
\hline 9. Palaua malvifolia Cavanilles & & $\mathrm{X}$ \\
\hline 10. Lippia nodiflora (L.) Michaux & & $\mathrm{X}$ \\
\hline $\begin{array}{l}\text { 11. Grindelia glutinosa (Cavanilles) } \\
\text { Dunal }\end{array}$ & $X$ & $\mathrm{X}$ \\
\hline $\begin{array}{l}\text { 12. Chenopodium macrospermun } \\
\text { Hooker } \mathrm{f} \text {. }\end{array}$ & $\mathrm{X}$ & $X$ \\
\hline $\begin{array}{l}\text { 13. Cotula australis (Sieber ex } \\
\text { Sprengel) Hooker f. }\end{array}$ & & $\mathrm{X}$ \\
\hline 14. Nasa urens (Jacq.) Weigend & & $\mathrm{X}$ \\
\hline 15. Dicliptera ruiziana Wasshausen & $\mathrm{X}$ & $\mathrm{X}$ \\
\hline $\begin{array}{l}\text { 16. Pennisetum clandestinum } \\
\text { Hochstetter ex Chiovenda }\end{array}$ & $X$ & \\
\hline $\begin{array}{l}\text { 17. Vasquezia oppositifolia (Lagasca) } \\
\text { S. F. Blake }\end{array}$ & $\mathrm{X}$ & \\
\hline $\begin{array}{l}\text { 18. Dichondra microcalyx (Hallier f.) } \\
\text { Fabris }\end{array}$ & $X$ & \\
\hline 19. Cyperus squarrosus L. & $\mathrm{X}$ & \\
\hline 20. Spergularia $\mathrm{sp}$. & $\mathrm{X}$ & \\
\hline 21. Marrubium vulgare L. & $\mathrm{X}$ & \\
\hline
\end{tabular}

Relación entre las condiciones meteorológicas y la sucesión vegetal

Sólo 4 de los 10 parámetros mostraron correlaciones significativas $(\mathrm{p}<0.05)$ con alguno de los parámetros meteorológicos (Tabla 2), siendo la captación de neblina el parámetro más relacionado a la dinámica vegetal observada durante el periodo Febrero 2005 - Febrero 2006. Existieron 5 correlaciones significativas con la captación de neblina, 2 con la temperatura media, y ninguna con la precipitación (Tabla 2).

Tabla 2. Correlaciones lineales entre los parámetros registrados en las parcelas de los cerros Barrera y Lagunillas, y las condiciones meteorológicas entre Febrero 2005 y Febrero 2006 en las Lomas de Atiquipa. (Los números en negritas indican correlaciones estadísticamente significativas).

\begin{tabular}{|c|c|c|c|c|}
\hline Parámetro & Ubicación & Temperatura & Neblina & $\begin{array}{l}\text { Precipi- } \\
\text { tación }\end{array}$ \\
\hline \multirow{4}{*}{$\begin{array}{l}\text { Individuos } \\
\text { total }\end{array}$} & Barrera & -0.2892 & 0.7892 & 0.4174 \\
\hline & $\mathrm{p}$ & 0.338 & 0.001 & 0.156 \\
\hline & Lagunillas & -0.1998 & 0.4995 & 0.2155 \\
\hline & $\mathrm{p}$ & 0.513 & 0.082 & 0.48 \\
\hline \multirow{4}{*}{$\begin{array}{l}\text { Cobertura } \\
\text { vegetal }\end{array}$} & Barrera & 0.0885 & 0.1624 & -0.1517 \\
\hline & $\mathrm{p}$ & 0.774 & 0.596 & 0.621 \\
\hline & Lagunillas & -0.11 & 0.2514 & -0.1519 \\
\hline & $\mathrm{p}$ & 0.721 & 0.407 & 0.62 \\
\hline \multirow{4}{*}{$\begin{array}{l}\text { Individuos } \\
\text { adultos }\end{array}$} & Barrera & 0.5045 & -0.4659 & -0.2333 \\
\hline & $\mathrm{p}$ & 0.079 & 0.109 & 0.443 \\
\hline & Lagunillas & -0.0046 & -0.0388 & -0.0418 \\
\hline & $\mathrm{p}$ & 0.988 & 0.9 & 0.892 \\
\hline \multirow{4}{*}{$\begin{array}{l}\text { Cobertura } \\
\text { adultos }\end{array}$} & Barrera & 0.3629 & -0.2801 & -0.3208 \\
\hline & $\mathrm{p}$ & 0.223 & 0.354 & 0.285 \\
\hline & Lagunillas & 0.0536 & 0.1104 & -0.1081 \\
\hline & $\mathrm{p}$ & 0.862 & 0.72 & 0.725 \\
\hline \multirow[t]{4}{*}{ Plántulas } & Barrera & -0.2975 & 0.7933 & 0.4199 \\
\hline & $\mathrm{p}$ & 0.324 & 0.001 & 0.153 \\
\hline & Lagunillas & -0.2839 & 0.7724 & 0.3646 \\
\hline & $\mathrm{p}$ & 0.347 & 0.002 & 0.221 \\
\hline \multirow{4}{*}{$\begin{array}{l}\text { Cobertura } \\
\text { plántulas }\end{array}$} & Barrera & -0.1422 & 0.4554 & 0.0125 \\
\hline & $\mathrm{p}$ & 0.643 & 0.118 & 0.968 \\
\hline & Lagunillas & -0.2088 & 0.4637 & -0.0849 \\
\hline & $\mathrm{p}$ & 0.494 & 0.111 & 0.783 \\
\hline \multirow{4}{*}{$\begin{array}{l}\mathrm{N}^{\circ} \\
\text { especies }\end{array}$} & Barrera & 0.5238 & -0.3582 & -0.403 \\
\hline & $\mathrm{p}$ & 0.066 & 0.23 & 0.172 \\
\hline & Lagunillas & -0.0684 & 0.3181 & -0.0872 \\
\hline & $\mathrm{p}$ & 0.824 & 0.29 & 0.777 \\
\hline \multirow[t]{4}{*}{ Simpson } & Barrera & -0.6072 & 0.6483 & 0.5373 \\
\hline & $\mathrm{p}$ & 0.028 & 0.017 & 0.058 \\
\hline & Lagunillas & -0.5888 & 0.5216 & 0.3329 \\
\hline & $\mathrm{p}$ & 0.034 & 0.068 & 0.266 \\
\hline \multirow[t]{4}{*}{ Shannon } & Barrera & 0.2998 & -0.7628 & -0.5323 \\
\hline & $\mathrm{p}$ & 0.32 & 0.002 & 0.061 \\
\hline & Lagunillas & 0.309 & -0.1649 & -0.42 \\
\hline & $\mathrm{p}$ & 0.304 & 0.59 & 0.153 \\
\hline \multirow[t]{4}{*}{ Hojarasca } & Barrera & 0.4309 & -0.221 & -0.3315 \\
\hline & $\mathrm{p}$ & 0.142 & 0.468 & 0.269 \\
\hline & Lagunillas & 0.3039 & -0.477 & -0.3482 \\
\hline & $\mathrm{p}$ & 0.313 & 0.099 & 0.244 \\
\hline
\end{tabular}


El número total de individuos por parcela registrados en las evaluaciones mensuales en Barrera y la variación en la captación de neblina mostraron una correlación significativa y buena $(\mathrm{r}=0.79 ; \mathrm{p}=0.001)$. La relación directamente proporcional entre estos parámetros se hizo más evidente en el periodo Setiembre 2005 - Febrero 2006, mientras que en el periodo Mayo 2005 - Julio 2005 no existió relación aparente entre estos parámetros (Figura 3).

El número de plántulas por parcela registradas en ambos cerros durante el periodo Febrero 2005 Febrero 2006 mostró una correlación positiva y significativa con la captación de neblina (Barrera $\mathrm{r}=0.79 ; \mathrm{p}=0.001$; Lagunillas $\mathrm{r}=0.77 ; \mathrm{p}=0.002$ ). Estas correlaciones son buenas, y al igual que en el caso del número total de individuos por parcela, se hicieron más evidentes a partir de Setiembre 2005 (Figura 4).

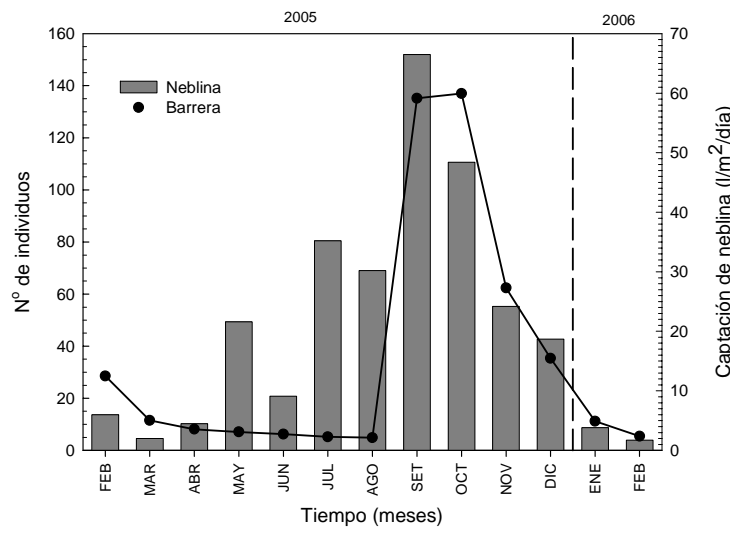

Figura 3. Relación entre la variación en el número total de individuos registrados en cerro Barrera y la variación en la captación de neblina entre Febrero 2005 y Febrero 2006. $(r=0.79 ; p=0.001)$.

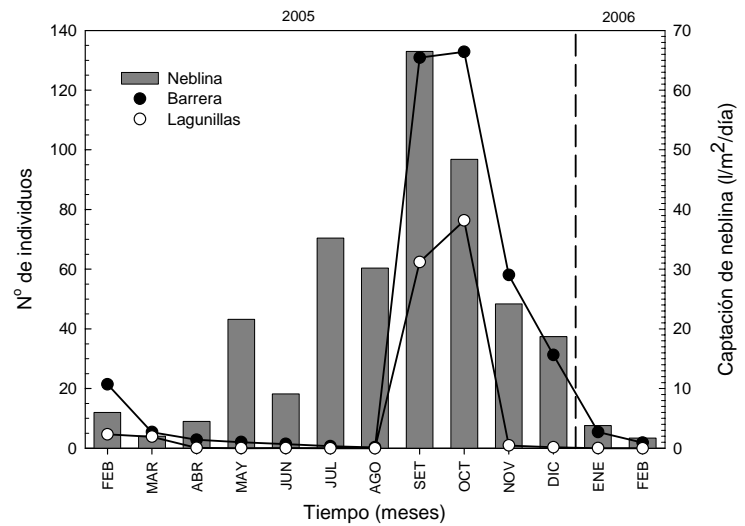

Figura 4. Relación entre la variación en el número de plántulas registradas en los cerros Barrera y Lagunillas y la variación en la captación de neblina entre Febrero 2005 y Febrero 2006. (Barrera $\mathrm{r}=0.79$; $\mathrm{p}=0.001$; Lagunillas $r=0.77 ; \mathrm{p}=0.002$ ).
En Barrera en comparación con Lagunillas, la variación mensual en el número de plántulas por parcela se ajustó mejor a la variación mensual en la captación de neblina. En Barrera la variación mensual en el predominio, medido a través del índice de Simpson, y la variación mensual en la captación de neblina mostraron una correlación significativa, buena y positiva $(\mathrm{r}=0.65 ; \mathrm{p}=0.017)$. El pico en la captación de neblina de Setiembre 2005 coincidió con el pico del predominio por parcela, cuando los valores de este último estuvieron muy próximos a 1 (Figura 5). En Lagunillas la correlación entre estos dos parámetros no fue significativa.

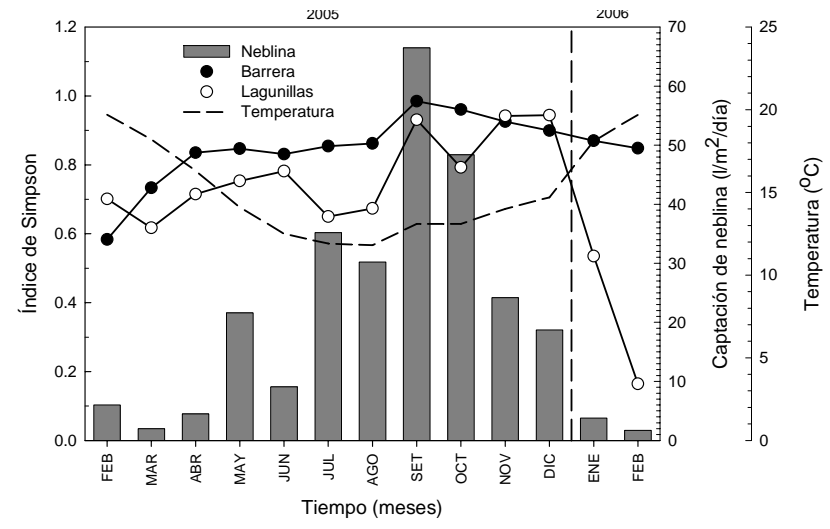

Figura 5. Relación entre la variación en los índices de Simpson calculados para los cerros Barrera y Lagunillas, la variación en la captación de neblina y la variación en la temperatura media entre Febrero 2005 y Febrero 2006. (Barrera-Neblina: $r=0.65$; $\mathrm{p}=0.017$; Barrera-Temperatura: $\mathrm{r}=-0.61 ; \mathrm{p}=0.028$; LagunillasTemperatura: $\mathrm{r}=-0.59 ; \mathrm{p}=0.034)$.

Para ambos cerros la variación mensual en la temperatura media y la variación mensual en el predominio, medido a través del índice de Simpson, mostraron una correlación buena, significativa, pero negativa (Barrera $\mathrm{r}=-0.61 ; \mathrm{p}=0.028$; Lagunillas $\mathrm{r}=$ $0.59 ; \mathrm{p}=0.034)$. En este caso la disminución de la temperatura estuvo relacionada a un incremento en el predominio en las parcelas, principalmente entre Junio 2005 y Setiembre 2005, cuando la temperatura media cayó por debajo de $14{ }^{\circ} \mathrm{C}$ (Figura 5). La variación en el predominio en Barrera mostró un mejor ajuste a la variación en la temperatura. El último parámetro que mostró correlación significativa buena $(\mathrm{r}=-0.76$; $\mathrm{p}=0.002$ ), fue la diversidad, medida a través del índice de Shannon-Wiener. En Barrera, se evidenció que durante meses con mayor captación de neblina, la diversidad en sus parcelas fue menor. Esta relación inversa se observó desde Julio 2005 hasta Febrero 2006, especialmente en Setiembre 2005 cuando la captación de neblina fue máxima y la diversidad mínima, muy próxima a 0 bits/ind. (Figura 6). 


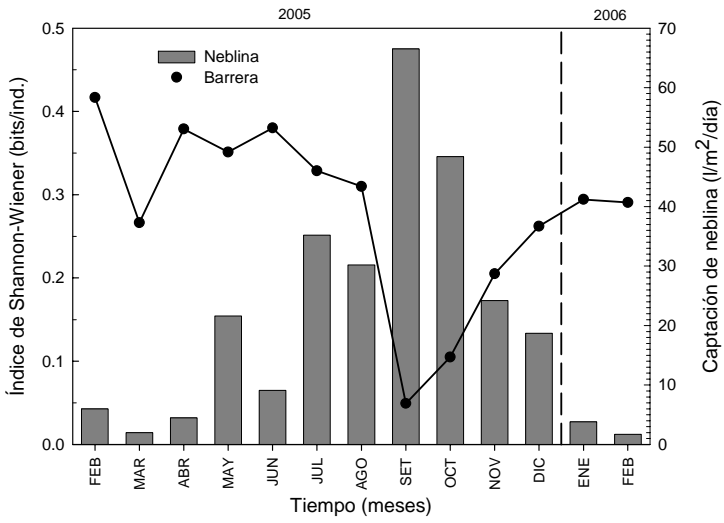

Figura 6. Relación entre la variación en los índices de Shannon-Wiener calculados para cerro Barrera y la variación en la captación de neblina entre Febrero 2005 y Febrero 2006. $(r=-0.76$; $p=0.002)$.

\section{Discusión}

Las correlaciones positivas entre la variación en el número total de individuos por parcela $\mathrm{y}$ el de plántulas por parcela en ambos cerros con la variación en la captación de neblina confirman el patrón neblina - dependiente de los ecosistemas de Lomas descrito por varios autores como Ono (1986), Rundel et al. (1991), Mostacero et al. (1996), Gutiérrez (2001), Thompson et al. (2003), entre otros. Dentro de estas correlaciones, la correlación entre la variación en el número total de individuos por parcela y la variación en la captación de neblina para Lagunillas no fue significativa, aunque su valor se acerque a la significancia (Tabla 2). Esto último pudo deberse a que la comunidad de Barrera produjo mayor cantidad de plántulas, y por lo tanto una mayor población total de individuos que la de Lagunillas, lo que hace que el patrón sea más distinguible mediante una prueba estadística.

Además, la buena correlación significativa existente entre la variación en el número de plántulas por parcela en Lagunillas y la variación en la captación de neblina puede explicarse por la naturaleza estacional de la vegetación de este cerro, debido a que la variación mensual en el número de plántulas por parcela refleja la variación de la comunidad; sin embargo esta relación entre el número de plántulas y el número total de individuos no llegó a la magnitud de efecto necesaria para que el número total de individuos y la captación de neblina muestren una correlación significativa, como en Barrera. Debemos destacar, también, que la comunidad de Barrera esta dominada por arbustos, como es el caso de $S$. mollendoensis y $N$. paniculata, los cuales son los principales captadores de neblina; mientras que Lagunillas tiene pocos arbustos.
La germinación masiva de semillas ocurrida en setiembre 2005 puede explicarse haciendo referencia al mecanismo de latencia propuesto por Vidiella \& Armesto (1989), Vidiella (1992) y Figueroa \& Jaksic (2004), llamado latencia innata y que vincula exclusivamente la respuesta del banco de semillas a los meses con condiciones ambientales favorables (temperatura e insolación bajas), propias del invierno en el desierto costero peruano - chileno. Este mecanismo impide la germinación de semillas por debajo de un umbral de precipitación, que estaría relacionado a la presencia de ciertas sustancias inhibidoras de germinación que deben ser lavadas por las lluvias antes de que las semillas logren germinar.

Ambos cerros presentaron diferencias, tanto en predominio, como en diversidad de Shannon-Wiener, aunque para ambos el predomino estuvo entre medio y alto, y la diversidad se mantuvo baja. Además, existió una buena correlación significativa, pero negativa entre el predominio y la temperatura media. Los incrementos en el número de individuos obedecen al incremento del recurso hídrico, manifestado en una mayor captación de neblina y precipitación, que a su vez están relacionadas a una disminución en la temperatura durante los meses de invierno. Esta última afirmación explica el hecho de haberse encontrado la correlación negativa ya mencionada.

El conjunto de datos que más se ajusta a la dinámica vegetal fue la captación de neblina, lo que indica que las comunidades de Lomas se generan bajo la influencia de las neblinas invernales, corroborando las afirmaciones de Pefaur (1982), Rundel et al. (1991), Mostacero et al. (1996) y Gutiérrez (2001). Sin embargo, la precipitación que es una variable climática citada en la literatura (Gutiérrez, 2001; Thompson et al., 2003) no resultó tener ninguna correlación significativa con los parámetros evaluados. Esta ausencia de correlaciones significativas puede deberse al hecho de que los estudios de Gutiérrez (2001) y Thompson et al. (2003) analizaban datos correspondientes a varios años, por lo tanto es posible que la corta escala temporal de este estudio esté escondiendo un efecto, que de analizarse más años debería estar presente. Esta última afirmación se puede apoyar en el hecho de que precipitación y captación de neblina presentaron patrones similares durante el periodo de estudio (Figura 2), pero que en el caso de la captación de neblina los registros son significativamente mayores a los de precipitación, lo que estaría evidenciando no una falta de efecto, sino tal vez una falta de poder en la prueba estadística. Incluso, al analizar la Figura 2 en relación a la emergencia masiva de plántulas en Setiembre 2005, podemos decir que ésta emergencia fue tal vez efecto de la precipitación acumulada entre Julio 2005 y Agosto 2005.

Durante este estudio la "época de Lomas" (mayor desarrollo de la vegetación) se extendió entre 
Setiembre 2005 hasta Diciembre 2005, aunque fue en Noviembre 2005 cuando se registraron los picos de desarrollo. Este resultado contrasta con lo reportado por Arias \& Torres (1990), debido a que estos autores establecen que la "época de Lomas" se desarrolló entre Setiembre y principios de Noviembre para el periodo Marzo 1988 - Marzo 1989. Este alargamiento de la época de Lomas observado en el periodo estudiado podría estar relacionado a la mayor cantidad de humedad que existió en este periodo en comparación al periodo que evaluaron Arias \& Torres (1990).

Los registros históricos de las condiciones meteorológicas para Atiquipa (CIZA et al., 1989), muestran un patrón claro para la temperatura media y precipitación en estas Lomas; así los meses entre Junio y Setiembre son los meses más fríos y húmedos, ya que la temperatura es menor y hay mayor cantidad de precipitación. Por otro lado, los meses entre diciembre y marzo son los meses más secos, con mayores temperaturas y menor cantidad de precipitación. Los datos de captación de neblina para el periodo Octubre 2002 - Agosto 2006 en Atiquipa (Proyecto PER/01/G35, datos no publicados), también confirman esta afirmación, siendo los meses entre Junio y Setiembre los más húmedos, y los meses entre Diciembre y Marzo los más secos. Esto permite establecer que, si bien es cierto no se dispuso de todos los datos meteorológicos para el periodo de estudio, los datos que fueron finalmente utilizados para el análisis de correlación lineal reflejan adecuadamente el patrón climático de las Lomas de Atiquipa, y por lo tanto otorgan mayor validez y repetitibilidad a los resultados encontrados a partir de este análisis.

Finalmente, el actual escenario de cambio climático que estaría modificando los regímenes de humedad en todo el planeta constituiría una amenaza muy importante para un ecosistema como el de Lomas, debido a que depende grandemente de la humedad del ambiente, ya sea en forma de precipitación o en forma de neblina. Esta amenaza junto con otras amenazas ya presentes, como la sobreexplotación del suelo, evidencian la necesidad de mayores estudios sobre la dinámica de estos frágiles ecosistemas costeros.

\section{Conclusiones}

Los resultados de este trabajo permiten apoyar la hipótesis de trabajo de que las Lomas de Atiquipa representan comunidades vegetales que se desarrollan bajo la influencia de neblinas invernales. Se encontró relación significativa entre dinámica vegetal $\mathrm{y}$ condiciones meteorológicas. La neblina fue la más relacionada al desarrollo de las comunidades vegetales en los cerros de estudio, y presentó una relación directa con el número total de individuos y la emergencia de plántulas, así como con el predominio; y de manera inversa con la diversidad de Shannon -
Wiener. Existió una relación inversa entre la temperatura y el predominio en la comunidad, de tal manera que menores temperaturas estuvieron relacionadas a mayor predominio. No se encontró relación significativa entre la precipitación natural y la dinámica vegetal, aunque se puede sugerir la existencia de un efecto acumulativo entre Julio y Agosto del 2005 que desencadenó el desarrollo de Setiembre 2005.

\section{Agradecimientos}

Este trabajo recibió financiamiento del Proyecto "Recuperación y Uso Sostenible de los Ecosistemas de las Lomas de Atiquipa y Taimara, por Gestión Comunal" (PER/01/G35). Los miembros de este Proyecto, así como todos los pobladores de la Comunidad Campesina de Atiquipa colaboraron en la fase de campo. Al Ing. Sebastián Zúñiga, Director del SENAMHI-Arequipa, y al Blgo. Edgar Gutiérrez por los datos meteorológicos de la Estación Punta Atico. DASM agradece el apoyo de la Beca CONICYT M58080091 durante la redacción final de este documento. Agradecemos los acertados comentarios y sugerencias de dos revisores anónimos.

\section{Literatura citada}

Arias C. \& Torres J. 1990. Dinámica de la vegetación de las Lomas del sur del Perú: Estacionalidad y Productividad Primaria, Caso: Lomas de Atiquipa (Arequipa). Zonas Áridas. 6: 55-76.

BOLFOR, Mostacedo B. \& Fredericksen T. 2000. Manual de Métodos Básicos de muestreo y análisis en Ecología Vegetal. Editora El País. Santa Cruz-Bolivia.

Braun Blanquet J. 1979. Fitosociología: Bases para el estudio de las comunidades vegetales. Blume Ediciones. Madrid-España.

Canziani J. \& Mujica E. 1997. Atiquipa: un caso prehispánico de manejo sustentable en ecología de Lomas. Páginas 503-526. En: E. González, B. Revesz \& A. Tapia (editores.) Perú: El problema agrario en debate. Seminario Permanente e Investigación Agraria VI. Lima-Perú.

CIZA, ONERN \& SENAMHI. 1989. Aprovechamiento de nieblas costeras en las zonas áridas de la costa, Lomas de Atiquipa (Prov. Caravelí, Dpto. Arequipa). CONCYTEC. Lima-Perú.

Engel F. 1981. Prehistoric Andean Ecology Man, Settlement and Environment in the Andes. The Deep South. University of New York. USA.

Ferreyra R. 1986. Flora y vegetación del Perú. Gran Geografía del Perú Volumen 2. Editorial Manfer-Juan Mejía Baca. España.

Figueroa J. \& Jaksic F. 2004. Latencia y banco de semillas en plantas de la región mediterránea de Chile central. Revista Chilena de Historia Natural.77(1): 201-215.

Gutiérrez J. 2001. Dynamics of ephemeral plants in the coastal desert of North-Central Chile. Páginas 105-124 en: I. Prakash (editor) Ecology of desert environments. Scientific Publishers. Jodhpur-India.

McCook L. 1994. Understanding ecological community succession: causal models and theories, a review. Vegetatio. 110: 115-147. 
Moreno C. 2001. Métodos para medir la biodiversidad. M \& T Manuales y Tesis SEA Volumen 1. Zaragoza-España.

Mostacero J., Mejía F. \& Peláez F. 1996. Fitogeografía del Norte del Perú. Serie Ciencias-CONCYTEC. LimaPerú.

Müller G. 1985. Zur floristischen Analyse der peruanischen Loma - Vegetation. Flora. 176: 153-165.

ONERN. 1976. Mapa Ecológico del Perú. Guía Explicativa. Oficina Nacional de Recursos Naturales. Lima-Perú.

Ono M. 1986. Definition, classification and taxonomic significance of the Lomas vegetation. Páginas 5-14 en: M. Ono (editor) Taxonomic and Ecological Studies on the Lomas Vegetation in the Pacific Coast of Peru. Makino Herbarium, Tokyo Metropolitan Univesity. Tokyo-Japan.

Péfaur J. 1982. Dynamics of plant communities in the Lomas of southern Perú. Vegetatio. 49: 163-171.

Rundel P., Dillon M., Palma B., Money H., Gulmon L. \& Ehleringer J. 1991. The phytogeography and ecology of the coastal Atacama and Peruvian deserts. Aliso. 13(1): $1-49$.
StatSoft Inc. 2001. STATISTICA for Windows, Version 6.0, Computer program manual. Tulsa, OK - USA. URL: http://www.statsoft.com

Thompson M., Palma B., Knowles J. \& Holbrook M. 2003. Multiannual climate in Parque Nacional Pan de Azúcar, Atacama Desert, Chile. Revista Chilena de Historia Natural. 76: 235-254.

Vidiella P. 1992. Desierto florido: Estudio experimental de la emergencia de plantas efímeras en respuesta a distintos regímenes de precipitación. Tesis de Magíster en Ciencias Biológicas. Universidad de Chile.

Vidiella P. \& Armesto J. 1989. Emergence of ephemeral plant species from soil samples of the Chilean coastal desert in response to experimental irrigation. Revista Chilena de Historia Natural. 62: 99-107.

Villegas L., Caballero K. \& Zeballos H. 2004. Rol ecológico de la avifauna de las Lomas de Atiquipa, Arequipa. Dilloniana. 4(1): 156-158.

\section{Anexo citado en el texto}

Anexo 1. Índices de Simpson y Shannon - Wiener de cada mes evaluado para los cerros Barrera y Lagunillas de las Lomas de Atiquipa entre Febrero 2005 y Febrero 2006.

\begin{tabular}{lllll}
\hline & \multicolumn{2}{c}{ Simpson } & \multicolumn{2}{c}{ Shannon - Wiener (bits/ ind.) } \\
\hline Febrero & Barrera & Lagunillas & Barrera & Lagunillas \\
Marzo & $0 . .5832$ & 0.7007 & 0.4166 & 0.6897 \\
Abril & 0.7335 & 0.6172 & 0.2663 & 0.8746 \\
Mayo & 0.8354 & 0.7148 & $0 . .3788$ & 0.4777 \\
Junio & 0.8468 & 0.7535 & $0 . .3511$ & 0.3423 \\
Julio & 0.831 & 0.7818 & $0 . .38$ & 0.2299 \\
Agosto & 0.8543 & 0.6499 & $0 . .3285$ & 0.1765 \\
Setiembre & 0.8619 & 0.6733 & $0 . .3097$ & 0.1233 \\
Octubre & 0.9848 & 0.9309 & $0 . .0493$ & 0.2015 \\
Noviembre & 0.9602 & 0.7927 & 0.105 & 0.5549 \\
Diciembre & 0.8993 & $0 . .9419$ & 0.2049 & 0.1625 \\
Enero & 0.8698 & $0 . .5349$ & 0.2944 & 0.1615 \\
Febrero & 0.8482 & 0.1645 & 0.2905 & 0.0477 \\
\hline Promedio & $\mathbf{0 . 8 4 8 8}$ & $\mathbf{0 . 7 0 7 7}$ & $\mathbf{0 . 2 7 9 8}$ & $\mathbf{0 . 3 1 3 3}$ \\
\hline
\end{tabular}

${ }^{1}$ Proyecto "Recuperación y Uso sostenible de los ecosistemas costeros de las Lomas de Atiquipa y Taimara, por Gestión Comunal (PER/01/G35)" y Laboratorio de Ecología. Departamento Académico de Biología. Universidad Nacional de San Agustín de Arequipa. Av. Alcides Carrión s/n, Arequipa - Perú. Dirección actual: Departamento de Biología, Universidad de La Serena, Casilla 599, La Serena - Chile. diego.sotomayor@gmail.com

${ }^{2}$ Proyecto "Recuperación y Uso sostenible de los ecosistemas costeros de las Lomas de Atiquipa y Taimara, por Gestión Comunal (PER/01/G35)” y Laboratorio de Ecología. Departamento Académico de Biología. Universidad Nacional de San Agustín de Arequipa. Av. Alcides Carrión s/n, Arequipa - Perú. pejimi@hotmail.com 